\title{
Presentación atípica de injuria aórtica por traumatismo cerrado de tórax
}

\author{
Atypical presentation of blunt thoracic aortic injury traumatic
}

Miguel Osvaldo Villegas' ${ }^{1}$, Jorge Gómez ${ }^{2}$, Marcela Liliana Valenzuela³, Federico Martín Riolo', Alberto Pérez Mereles ${ }^{4}$

\section{RESUMEN}

El traumatismo cerrado de tórax con injuria aórtica es la segunda causa de muerte por traumatismo. El 80-85\% de los pacientes fallecen antes de llegar a un centro de salud, el resto necesita un diagnóstico rápido para realizar un tratamiento eficaz. La mayoría de los casos se dan por una desaceleración brusca, generalmente por accidentes de tránsito.

Presentamos un caso de traumatismo cerrado de tórax por aplastamiento debido a un accidente de tránsito que al año presenta hipertensión arterial, con estudios diagnósticos que informan dilatación de la aorta torácica descendente y una membrana en la luz con comportamiento estenótico. Se realizó tratamiento endovascular con endoprótesis aórtica balón expandible.

Palabras claves: aorta torácica, ruptura traumática, injuria cerrada, ruptura aórtica.

\section{ABSTRACT}

Blunt thorax trauma with aortic injury is the second cause of death for trauma. 80$85 \%$ of patients die before reaching a healthcare, the rest of the patients need a rapid diagnosis to perform and effective treatment. Most case are due to a sudden deceleration, usually due to traffic accidents.

We present a case of traffic accident with blunt thorax trauma by crushing that the year presented arterial hypertension, with angio-CT that report the presence of dilatation of the thoracic aorta with a membrane in the lumen of the artery and stenotic behavior. Endovascular treatment was done with aortic device expandible ballon.

Key words: thoracic aorta, traumatic rupture, blunt injuries, aortic injuries.

Revista Argentina de Cardioangiología Intervencionista 2018;9(2):97-99. Doi: 10.30567/RACI/201802/0097-0099

\section{INTRODUCCIÓN}

El traumatismo cerrado de tórax es la segunda causa de muerte traumática luego del traumatismo de cráneo, que puede ser dado por impacto directo sobre el tórax o por desaceleración, provocando injuria de múltiples órganos ${ }^{1}$. La mayoría de los pacientes que presentan un traumatismo cerrado de tórax no llegan a un centro de atención; quienes lo hacen y reciben un diagnóstico y tratamiento rápido suelen presentar buena evolución ${ }^{2}$.

Presentamos el caso de un paciente con injuria de aorta torácica descendente que evolucionó con leve dilatación aórtica e hipertensión arterial (HTA) por presencia de una membrana en la luz de la aorta con comportamiento estenótico.

\section{CASO CLÍNICO}

Paciente de 21 años con antecedente de haber sufrido un accidente de tránsito con aplastamiento de tórax un año atrás, con presencia de hemotórax, fractura de esternón y costillas siendo intervenido quirúrgicamente.

Al año presenta HTA diagnosticada en un control prelaboral, por lo cual recibe tratamiento con tres drogas (IECA, diuréticos y betabloqueantes) con mala respuesta. Se sospe-

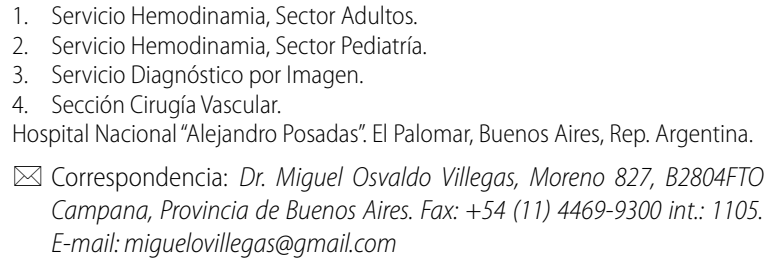

cha la presencia de una coartación de aorta y es evaluado por Hemodinamia Pediátrica, que solicita una angio-TAC donde se observa dilatación segmentaria de aorta torácica descendente (Figura 1A) e imagen compatible con injuria de la pared aórtica observado en la reconstrucción 3D (Figura 1B). Un aortograma confirmó la presencia de una membrana en la luz con gradiente de $60 \mathrm{mmHg}$ comportándose como una pseudocoartación de aorta (Figuras 2A y 2B). Se decide realizar tratamiento endovascular con implante de endoprótesis torácica balón expandible de $25 \times 150 \mathrm{~mm}$ (SETA RAK, Latecba. Buenos Aires, Argentina) (Figura 3A). La decisión de utilizar una prótesis balón expandible se toma por desconocerse la rigidez de la membrana, asegurarnos de que la misma quede normoimplantada y no tener complicaciones ante una eventual ruptura aórtica con extravasación de sangre al mediastino. En la angiografía control se ob-
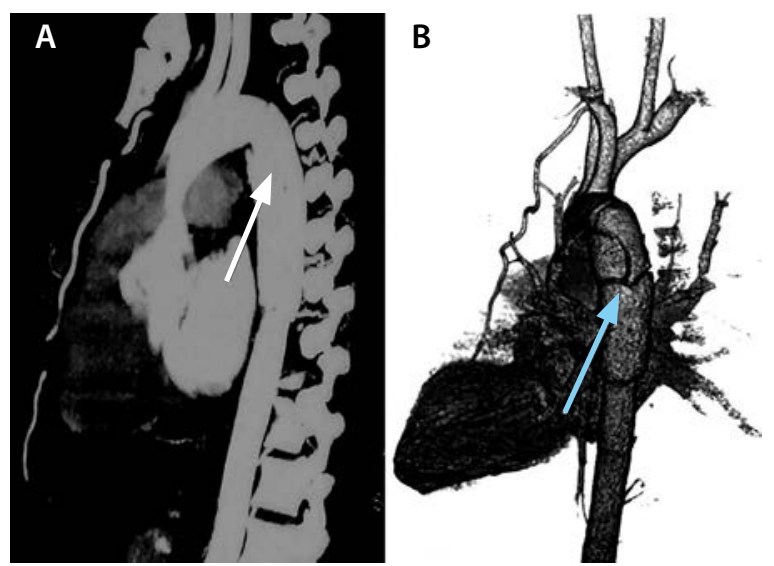

Figura 1. Angio-TAC. A. En un corte transversal se observa dilatación segmentaria de aorta descendente (flecha blanca). B. En la reconstrucción 3D se observa la extensión de la injuria aórtica (flecha celeste). 


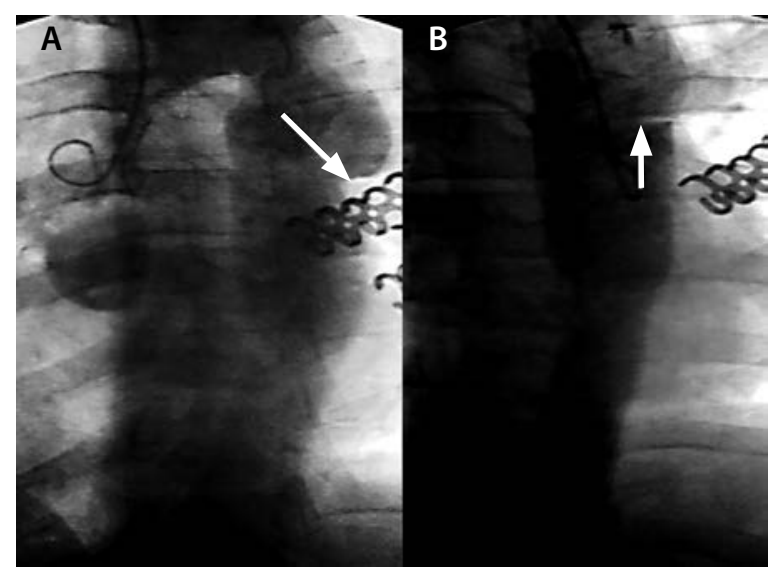

Figura 2. Aortograma basal. A y B. Se observa la presencia de una imagen compatible con membrana luminal en aorta descendente (flechas blancas) generando un gradiente de $60 \mathrm{mmHg}$.

serva endoleak Tipo I y se sobreexpande con balón de 30 × 30 mm (BALT ${ }^{\circ}$ Montmorency, Francia) (Figura 3B). En el último control se ve la presencia de una endoprótesis torácica normoimplantada y sin presencia de endoleak (Figura 3C).

$\mathrm{El}$ paciente evolucionó favorablemente, sin complicaciones, sin gradiente intraaórtico. En el seguimiento al año se presenta normotenso y sin medicación antihipertensiva.

\section{DISCUSIÓN}

El $80-85 \%$ de los pacientes que sufren un traumatismo cerrado de tórax fallecen antes de llegar a un centro de atención ${ }^{3}$. Los pacientes que llegan al centro de salud generalmente tienen ruptura contenida por la capa adventicia o las estructuras mediastinales vecinas ${ }^{4}$. En un estudio retrospectivo se observó que el traumatismo de tórax está acompañado en el $31 \%$ de los casos por injuria craneana y en el $29 \%$ por injuria abdominal5. El 30\% de los pacientes con injuria de aorta no presentan signos externos de traumatismo torácico y el $75 \%$ tienen fractura de costillas ${ }^{4}$.La muerte intrahospitalaria en pacientes que no reciben el tratamiento adecuado es del $30 \%$ dentro de las primeras 6 horas, el $40-50 \%$ dentro de las 24 horas y $90 \%$ dentro de los 4 meses $^{6}$. El caso que se presenta tiene una evolución mayor al año hasta la realización del tratamiento adecuado.

Si bien es difícil conocer los mecanismos que producen la injuria aórtica, el riego más importante es la desaceleración brusca. Un estudio dice que el $74 \%$ de los casos son por accidentes automovilisticos ${ }^{7}$. Otra causa es la caída desde la altura $^{6}$. En una revisión sobre 142 autopsias se observó un desplazamiento oblicuo lateral del tórax con movimientos de compresión y estiramiento de la aorta a nivel del istmo provocando una laceración de la misma ${ }^{8}$.

Goarin et al. clasificaron las lesiones de aorta en 3 grados:

1ro: Lesión limitada a la capa intima de la arteria (flap intimal) o hematoma intramural.

2do: Lesión que compromete la capa íntima y la adventicia (pseudoaneurisma, hemomediastino).

3ro: Compromete las tres capas de la arteria con una rápida extravasación de sangre a los tejidos mediastínicos vecinos que amortiguan el sangrado.

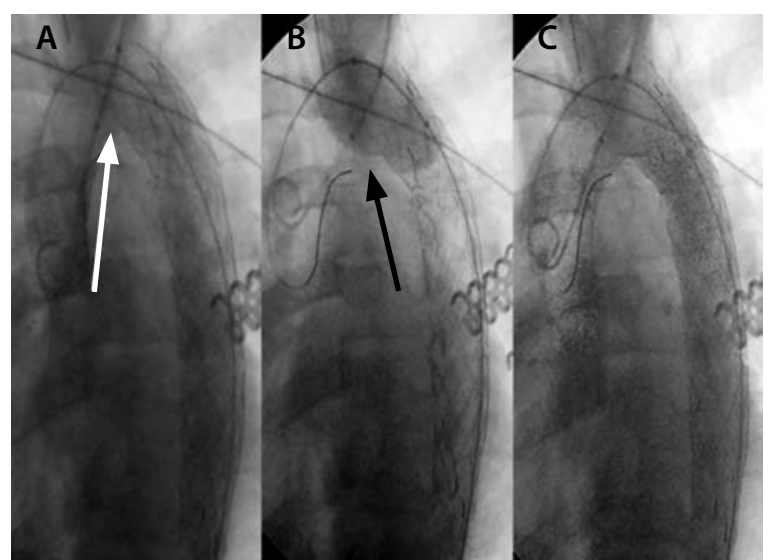

Figura 3. Radioscopia y angiografía del implante de la endoprótesis. A. Posimplante de endoprótesis (ZETA RAK, Latecba. Buenos Aires, Argentina). Se observa la presencia de endoleak en el extremo proximal (flecha blanca). B. Posdilatación con catéter balón de 30 × 30 mm (BALT. Montmorency, Francia) (flecha negra). C. Aortograma final. Se observa la prótesis bien implantada y ausencia de endoleak periprotésico.

Las lesiones de 3er grado se observan en el $40 \%$ de los pacientes ${ }^{6}$.

Los pacientes con traumatismo de tórax pueden presentar en la radiografía de tórax un ensanchamiento del mediastino, una radiografía normal no descarta una laceración aórtica, por lo que se debe realizar un ecocardiograma transesofágico y/o una tomografía computada de tórax con reconstrucción angiográfica'. El gold standard para el diagnóstico de la injuria de aorta es la angiotomografía computada que presenta una sensibilidad del $98 \%$ y una especificidad del $100 \%{ }^{6}$. La arteriografía tiene una alta sensibilidad y especificidad mayor al $90 \%$ para las lesiones grado 2 do y $3 \mathrm{ro}^{6}$. El paciente que presentamos tenía una lesión de 2 do grado.

El tratamiento frente a una injuria aórtica debe ser urgente. Existen varias formas de tratarlo; se puede realizar abordaje quirúrgico convencional con sutura directa de la lesión, resección del segmento afectado con interposición de prótesis de dacrón o el tratamiento endovascular, que actualmente es considerado de primera elección, con implante de endoprótesis torácica ${ }^{10}$.

Estudios comparativos entre el tratamiento endovascular y el quirúrgico convencional demostraron que el primero presenta un menor índice de lesión medular (3 vs. 9\%), menos trastornos renales (5vs. 8\%), menor mortalidad (9vs. 19\%) y menos complicaciones a largo plazo ${ }^{10}$.

El nuestro es un caso de presentación atípica, en el que la injuria aórtica del paciente es diagnosticada como consecuencia del estudio de su HTA después de un año de haber sufrido un traumatismo de tórax.

\section{CONCLUSIÓN}

La injuria de aorta puede pasar desapercibida, si esta no es sospechada desde un inicio y diagnosticada al momento del traumatismo. Este caso corresponde a una presentación atípica por su forma de presentación, el tiempo de evolución y el tratamiento elegido. 


\section{BIBLIOGRAFÍA}

1. Fabian TC, Richardson JD, Croce MA. Prospective study of blunt aortic injury: Multicenter Trial of the American Association for the Surgery of Trauma. J Trauma 1997 Mar;42(3):374-80; discussion 380-3.

2. Osgood MJ, Heck JM, Rellinger EJ, et al. Natural history of grade I-Il blunt traumatic aortic injury. J Vasc Surg 2014;59(2):334-41.

3. Sevitt S. The mechanisms of traumatic rupture of the thoracic aorta. Br J Surg 1977Mar;64(3):166-73.

4. Parmley LF, Mattingly TW, Manion WC, Jahnke EJ Jr. Non penetrating traumatic injury of the aorta. Circulation 1958 Jun;17(6):1086-101.

5. Arthurs ZM, Starnes BW, Sohn VY, Singh N, Martin MJ, Andersen CA. Functional and survival outcomes in traumatic blunt thoracic aortic injuries: an analysis of the National Trauma Databank. J Vascular Surg.2009 Apr;49(4):988-94.

6. Ait Ali Yahia D, Bouvier A, Nedulco C, et al. Imaging of thoracic aortic injury. Diagn Interven Imaging. 2015 Jan;96(1):79-88.
7. Cook J, Salerno C, Krishnadasan B, Nicholls S, Meissner M, Karmy-Jones R. The effect of changing presentation and management on the outcome of blunt rupture of the thoracic aorta. Journal of Thoracic and Cardiovascular Surgery.2006;131(3):594-600.

8. Feczko FD, Lynch L, Pless JE, Clark MA, McClain J, Hawley DA. An autopsy case review of 142 non penetrating (blunt) injuries of the aorta. The Journal of Trauma. 1992;33(6):846-9.

9. Demetriades D, Velmahos GC, Scaleaetal TM. Diagnosis and treatment of blunt thoracic aortic injuries: changing perspectives. The Journal of Trauma 2008;64(6):1415-8, 2008, discussion 1418-9.

10. LeeWA, Matsumura JS, Mitchell RS, et al. Endova scular repair of traumatic thoracic aortic injury: Clinical practice guidelines of the society for vascular surgery. J Vasc Surg 2011 Jan;531(1):187-92. 